\title{
Enhancing microaerobic plasmid DNA production by chromosomal expression of Vitreoscilla hemoglobin in E. coli
}

\author{
Karim Jaén ${ }^{1}$, Daniela Velazquez ${ }^{1}$, Juan Sigala Alanis², and Alvaro Lara ${ }^{1}$ \\ ${ }^{1}$ Universidad Autónoma Metropolitana \\ ${ }^{2}$ Universidad Autónoma Metropolitana-Cuajimalpa
}

April 28, 2020

\begin{abstract}
Oxygen availability and overflow metabolism are often limiting factors in high cell-density cultures. In the present study, expression of Vitreoscilla hemoglobin in the chromosome of Escherichia coli was used as a strategy to improve plasmid DNA (pDNA) production in biphasic fed-batch cultures. During the fully aerobic batch phase, the strain expressing VHb accumulated $28 \%$ less acetate and $19 \%$ more pDNA than the non-expressing strain. The fed-batch phase was carried out with a change of regime from aerobic to microaerobic conditions. The pDNA yields from biomass increased consistently in the VHb-expressing strain during the whole culture, while decreased progressively for the non-expressing strain during microaerobic conditions. The ratio of positive/negative plasmid replication control molecules (RNAII/RNAI) were lower for the VHb-expressing than for the non-expressing strain. However, the final pDNA titer was ca. $74 \%$ higher for the former. Flux balance analysis suggests that $\mathrm{VHb}$ presence increases the flux in anaplerotic pathways. The higher lactate production observed in VHb-expressing cells may be triggered by an increased demand of NAD+ in glycolysis under microaerobic conditions. These results are valuable for faster development of robust pDNA production processes.
\end{abstract}

\section{INTRODUCTION}

Plasmids are an essential tool for molecular biology and industrial biotechnology. In addition to applications like large-scale transient transfection, plasmid DNA (pDNA) is gaining relevance as a therapeutic agent. According to a recent survey, pDNA is used as the active pharmaceutical ingredient in most of all the gene therapy drugs (36.36 \%) approved worldwide from 1998 to 2019 (Ma et al., 2020). A pDNA vaccine against COVID-19 that express the SARS-CoV-2 spike is currently being evaluated in pre-clinical trials (Ahn et al., 2020). Therefore, efficient culture processes are needed for large-scale pDNA production. Efficient largescale processes require the availability of cell factories less sensitive to environmental fluctuations (Wehrs, Tanjore, Eng, Lievense, Pray, \& Mukhopadhyay, 2019). For instance, pDNA is usually produced in fedbatch, high cell-density cultures ofEscherichia coli (E. coli) (Rozkov, Gillström, Björnestedt, \& Schmidt, 2008). In high cell-density cultures, the oxygen demand by cells easily surpasses the oxygen transfer capacity of the bioreactor, which leads to local or general microaerobic conditions in the vessel (Palomares, Lara, \& Ramírez, 2012). $\mathrm{O}_{2}$ limitation in E. coli cultures is highly undesirable, since it triggers metabolic shifts that result in the production of organic acids, as well as loss of energy and biomass yields. Nevertheless, cultures under $\mathrm{O}_{2}$ limitation may allow to reach the maximum oxygen transfer rate $\left(\mathrm{OTR}_{\max }\right)$ capacity of the bioreactor at the operating power input, which is an economic advantage. In addition, it has been demonstrated that $\mathrm{O}_{2}$ limitation can increase the pDNA yields ( $\mathrm{Y}_{\mathrm{pDNA} / \mathrm{x}}$ ) (Passarinha et al., 2006; Lara, Jaén, Folarin, Keshavarz-Moore, \& Büchs, 2019). Therefore, microaerobic pDNA production can be an attractive technology, provided that strains that can better cope with $\mathrm{O}_{2}$ limitation are available. There have been recent efforts to develop $E$. coli strains with a superior performance under microaerobic conditions (Veeravalli et al., 2018; Jaén, Velazquez, Delvigne, Sigala, \& Lara 2019). A K-12-derived E. coli strain 
expressing Vitreoscilla hemoglobin ( $\mathrm{VHb}$ ) has been proposed as an attractive host to produce pDNA (Jaén, Velazquez, Delvigne, Sigala, \& Lara 2019). VHb is a well-known globin with a remarkable affinity for $\mathrm{O}_{2}$ and a fast delivery of it to the terminal oxidases. The application of $\mathrm{VHb}$ to improve microaerobic processes is well documented (Stark, Pagilla, \& Dikshit, 2015). However, its expression is usually plasmid-based, which is undesirable for pDNA production. Jaén and coworkers (2019) inserted the vgb gene, coding for VHb, under transcriptional control of $\mathrm{P}_{t r c}$ in the chromosome of $E$. coli W3110recA - The construction was integrated into the chromosome between lacI and lacZ by homologous recombination, making $v g b$ expression constitutive. The recA gene was inactivated to increase plasmid stability (Roca \& Cox, 1997). A positive effect on pDNA supercoiled fraction was also observed in the recA mutant, compared with its wild-type (Jaén, Velazquez, Delvigne, Sigala, \& Lara 2019). In the present contribution, both strains, W3110recA and W3110rec $A^{-} v g b^{+}$were cultured in mineral medium with glucose as carbon source to produce plasmid pUC18 in fed-batch mode. To evaluate the suitability of the engineered strain to produce pDNA under $\mathrm{O}_{2}$ limitations, microaerobic conditions were created in the fed-batch phase by decreasing the stirrer speed. Figure 1 shows the profiles of the cultures. Both strains depleted the initial glucose in ca. $3.5 \mathrm{hr}$. The fedbatch phase started at that time and the glucose concentration remained close to zero during the rest of the process (Figures 1a and 1e). The culture was maintained aerobic until $6 \mathrm{hr}$, then the dissolved oxygen (DOT) was set to $2 \%$ air sat. (Figures $1 \mathrm{~d}$ and $1 \mathrm{~h}$ ) and controlled via the stirrer speed. During the aerobic part of the fed-batch, both strains grew at rates approximately equal to the programmed dilution rate $(0.15 \pm 0.01$ and $0.13 \pm 0.01 \mathrm{hr}^{-1}$ for W3110rec $A^{-}$and W3110rec $A^{-} v g b^{+}$, respectively). During the microaerobic phase, strain W3110 rec $A^{-}$accumulated more biomass than $\mathrm{W}^{-} 3110 \mathrm{rec} A^{-}{ }^{-} \mathrm{vg} b^{+}$, reaching $29.5 \pm 4.0$ and $22.5 \pm 0.2$ $\mathrm{g} / \mathrm{L}$, respectively (Figures 1a and 1e). These results contrast with many studies that report an increased growth rate under microaerobic conditions when VHb is expressed (see Stark, Pagilla, \& Dikshit, 2015). However, the effect of $\mathrm{VHb}$ has been more frequently studied in batch cultures, where glucose was in excess. Enayati and co-workers (1999) reported higher biomass accumulation of E. coli JM103 expressing VHb from a high copy-number plasmid in fed-batch cultures in M9 medium, compared with the non-expressing cells. However, during the fed-batch cultures, those authors showed that the intracellular content of VHb reached more than $50 \mathrm{nmol} / \mathrm{g}_{\mathrm{WCW}}$, while in the strain used here, the amount of active $\mathrm{VHb}$ is only $1 \mathrm{nmol} / \mathrm{g}_{W C W}$ in batch cultures (unpublished data). The lower biomass accumulated by W3110recA ${ }^{-} v g b^{+}$, compared with W3110recA - could be originated by a higher burden due to the increase of plasmid replication and the consequent increased expression of bla (the gene conferring ampicillin resistance) (Figures 1c and $1 \mathrm{~g}$ ). During the batch phase, acetate was the most important by-product, the presence of VHb resulted in $c a .25$ $\%$ less acetate produced (Figures $1 \mathrm{~b}$ and $1 \mathrm{f}$ ). This is consistent with previous observations on the effect of $\mathrm{VHb}$ on overflow metabolism (Pablos, Sigala, Le Borgne, \& Lara, 2014). At the end of the process, the most highly accumulated by-product was lactate for both strains, although the amount accumulated by strain W3110rec $A^{-} v g b^{+}$was 5-fold higher than that of W3110recA - (Figures 1b and 1f) may be due an increased demand of $\mathrm{NAD}^{+}$. In fact, strain W3110rec ${ }^{-} \mathrm{vgb}{ }^{+}$accumulated more by-products than W3110 recA ${ }^{-}$. pDNA production profile is depicted in Figures $1 \mathrm{c}$ and $1 \mathrm{~g}$. The pDNA titer increased through the culture of W3110rec - $^{-}$The pDNA yield from biomass $\left(\mathrm{Y}_{\mathrm{pDNA} / \mathrm{X}}\right)$ remained around $3.5 \mathrm{mg} / \mathrm{g}$ during the batch phase and then increased to a maximum of $6.2 \pm 0.5 \mathrm{mg} / \mathrm{g}$ after $8 \mathrm{~h}$ of culture, to decrease to $5.4 \pm 1.1 \mathrm{mg} / \mathrm{g}$ at the end of the process (Fig. 1c). Although the supercoiled pDNA fraction (SCF) was higher than $85 \%$ during the whole culture, there was a trend to decrease after the transition to microaerobic conditions. At approximately $10 \mathrm{hr}$, the stirrer reached its maximum speed $(2000 \mathrm{rpm})$ and the DOT fell to $0 \%$ air sat. The $\mathrm{OTR}_{\text {max }}$ attained was $117 \mathrm{mmol} / \mathrm{L} \mathrm{hr}$ (Figure $1 \mathrm{~d}$ ). The maximum pDNA concentration was obtained at the end of the process, reaching $156.2 \pm 10.5 \mathrm{mg} / \mathrm{L}$ (Figure 1d). The performance of strain W3110recA ${ }^{-} \mathrm{vgb}{ }^{+}$for pDNA production was superior to that of $\mathrm{W} 3110$ rec ${ }^{-}$. The $\mathrm{Y}_{\mathrm{pDNA} / \mathrm{X}}$ in cultures of this $\mathrm{VHb}$ expressing strain remained around $4.5 \mathrm{mg} / \mathrm{g}$ during the batch phase and steadily increased, even after the transition to microaerobic conditions. The $\mathrm{Y}_{\mathrm{pDNA} / \mathrm{x}}$ reached a maximum of $12.1 \pm 0.3 \mathrm{mg} / \mathrm{g}$ at the end of the process (Figure 1g), more than the double of the yield reached by the non-expressing strain. Accordingly, the pDNA concentration reached a maximum of $271.6 \pm 4 \mathrm{mg} / \mathrm{L}$ at the end of the culture, which is $70 \%$ higher than that of the strain W3110recA - Similar to cultures of W3110recA -, DOT fell to $0 \%$ air sat. around $10 \mathrm{hr}$, and the $\mathrm{OTR}_{\max }$ was $125 \mathrm{mmol} / \mathrm{L} \mathrm{h}$ (Figure 1h). Moreover, the SCF remained above $95 \%$ 
regardless the $\mathrm{O}_{2}$ availability.

The performance of the strains was also investigated at molecular and metabolic levels. Samples were taken at 4, 6 and $12 \mathrm{hr}$ of culture to determine the plasmid copy number by chromosome (PCN, relative to theihfB gene) (Figure 2A). The results are in close agreement with the observed $\mathrm{Y}_{\mathrm{pDNA} / \mathrm{X}}$. For strain W3110recA ${ }^{-}$, the PCN was $c a .90$ at 4 and $10 \mathrm{hr}$, and increased to $178 \pm 11$ at $12 \mathrm{hr}$. For the strain W3110rec $A^{-} v g b^{+}$, the PCN increased from $182 \pm 69$ at $4 \mathrm{hr}$ to $556 \pm 126$ at the end of the process. The PCN of pUC plasmids is mainly controlled by two RNA molecules. Namely, RNAII serves as a primer to bind to the DNA and start the replication, while RNAI interacts with RNAII to inhibit the replication (del Solar, \& Espinosa, 2000). Therefore, increasing the intracellular RNAII copies/RNAI copies ratio is a strategy to increase the PCN (Freudenau, et al., 2015; Jaén, Velázquez, Sigala, \& Lara, 2019). Figure 2b depicts the RNAII copies/RNAI copies ratio from the different strains and sampling times, normalized to the sample at $2 \mathrm{hr}$. The RNAII copies/RNAI copies ratio increased in strain W3110rec $A^{-}$, at 10 and $12 \mathrm{hr}$, relative to $4 \mathrm{hr}$, which coincides with the measured $Y_{\mathrm{pDNA} / \mathrm{X}}$ (Figure 1c). In turn, the RNAII copies/RNAI copies ratio decreased to one-half at $10 \mathrm{hr}$ and increased to the same value than that at $2 \mathrm{hr}$ in strain W3110recA ${ }^{-} v g b^{+}$. This contrast with the PCN and measured $\mathrm{Y}_{\mathrm{pDNA} / \mathrm{X}}$ (Figure 1g and 2a), and implies that the PCN is also determined by the synthetic capacity of the host, as previously studied (Jaén, Velázquez, Sigala, \& Lara, 2019). The copy number of $v g b$ mRNA was also measured in W3110rec $A^{-} v g b^{+}$, and results relative to ihfB copies per chromosome normalized to the sample at $4 \mathrm{hr}$ are shown in Figure 2c. The $v g b$ copies decreased $c a .75 \%$ at $10 \mathrm{hr}$, but increased to ca. $9.1 \pm 1.5$ copies at the end of the culture. The latter increase could be attributed to a slower biomass formation rate during the last hours of the culture, in combination with the constitutive nature of the promoter. That results suggest that the $\mathrm{VHb}$-expressing strain can address more energy and building blocks to synthesize pDNA. This was further investigated by flux balance analysis (FBA). Figure 3 shows the estimated carbon fluxes in three pseudo steady-states: the exponential growth phase during the batch phase, and the aerobic and microaerobic phase during the fed-batch. During the batch phase, the specific glucose uptake rate $\left(q_{S}\right)$ was $13 \%$ higher for W3110rec $A^{-} v g b^{+}$, compared with W3110rec $A^{-}$. This was accompanied by a $13 \%$ increase on the specific $\mathrm{O}_{2}$ consumption rate $\left(q_{O 2}\right)$ and an $18 \%$ increase of the specific $\mathrm{CO}_{2}$ production rate. This implies that the carbon source can be oxidized faster when VHb is present under aerobic conditions. VHb expression increased the flux to the pentose phosphate pathway (PPP) and to ribose 5-phosphate (R5P) by 15 and $16 \%$, respectively, in comparison with the non-expressing strain. This may be a reason for the higher $\mathrm{Y}_{\mathrm{pDNA} / \mathrm{X}}$ observed in the batch phase in $\mathrm{W} 3110 \mathrm{rec} A^{-} v g b^{+}$, compared with W3110recA - (Figure 1). The flux from acetyl Co-A (AcCoA) to citrate (CIT) and from phosphoenol pyruvate (PEP) to oxaloacetate (OAA) were 20 and $10 \%$ higher in W3110recA ${ }^{-} v g b^{+}$, compared with W3110rec $A$ - The presence of VHb increased the flux from ICIT to $\alpha$-ketoglutarate (AKG) $c a$. $8 \%$, and the flux from malate (MAL) to oxaloacetate (OAA) by $26 \%$. In both reactions, NADH is produced. This cofactor is key for aerobic respiration and oxidative phosphorylation, and its estimated production rate is in agreement with the observed $q_{O 2}$ increase when $\mathrm{VHb}$ is expressed. During the aerobic glucose limitation phase, $q_{S}, q_{\mathrm{O} 2}$ and $q_{\mathrm{CO} 2}$ were similar, regardless the presence of $\mathrm{VHb}$ while during the batch phase, around $36 \%$ and $38 \%$ of glucose 6-phosphate was used in the PPP in W3110recA -and W3110recA $-v g b^{+}$, respectively. This fraction increased to $c a$. $59 \%$ in both strains during the aerobic part of the fed-batch, which may explain the $\mathrm{Y}_{\mathrm{pDNA} / \mathrm{X}}$ increase during this part of the process, compared to the batch phase (Figure 1). The estimated glycolytic fluxes were similar for both strains and the rates of by-products accumulation was negligible. Still, the flux from PEP to OAA was higher in W3110recA- $v g^{+}$, compared with W3110recA. The increased flux from ICIT to MAL due to VHb presence during the batch phase were decreased by $30 \%$ in W3110rec $A^{-} v g b^{+}$compared to W3110rec $A^{-}$during the aerobic part of the fed-batch. During the microaerobic fed-batch phase, $q_{S}$ increased by $27 \%$ in both strains with respect to the previous aerobic conditions. Both, $q_{\mathrm{O} 2}$ and $q_{\mathrm{CO} 2}$ were $c a .16 \%$ higher when $\mathrm{VHb}$ was expressed, consistent with the proposed action of this globin. However, the flux of G6P to the PP wasca. $11 \%$ higher in W3110recA - vgb ${ }^{+}$, compared with W3110rec $A^{-}$. DNA supercoiling requires the action of ATP-consuming gyrases (Cullis, Maxwell, \& Weiner; 1992). Therefore, the increased ATP generation expected from a higher $q_{O 2}$ may explain the higher SCF observed, particularly during the microaerobic phase in strain W3110recA ${ }^{-} v g b^{+}$(Figure 1). The glycolytic fluxes were between 5-12\% higher when VHb was expressed. Such glycolytic activity requires 
a higher $\mathrm{NAD}^{+}$supply, which may partially explain the considerably higher lactate synthesis by W3110rec $A$ ${ }^{-} v g b^{+}$, compared with W3110recA ${ }^{-}$. This hypothesis implies that the increased $q_{O 2}$ in W3110recA ${ }^{-} v g b^{+}$ is not enough to provide the necessary $\mathrm{NAD}^{+}$. In general, $\mathrm{W} 3110 \mathrm{rec} A^{-} v g b^{+}$produced more organic acids than W3110rec $A^{-}$. Frey and co-workers (2001) also reported an increase of lactate and succinate production when VHb was present in microaerobic cultures of MG1655. However, Tsai and co-workers (1996) found that when $\mathrm{VHb}$ was expressed in $\mathrm{W} 3110$, the synthesis of all the mixed-acid fermentation products was decreased. It is possible that such contradictory results may be related not only to the genetic background, but also to the amount of active $\mathrm{VHb}$ present. In the present experiments, the flux from AcCoA to CIT was only $5 \%$ higher when $\mathrm{VHb}$ was present, but the flux from PEP to OAA was 5-fold higher, compared with W3110rec $A^{-}$. The flux from ICIT to AKG was $50 \%$ lower, and the flux from MAL to OAA was only 9 $\%$ higher for the strain expressing $\mathrm{VHb}$, compared with the non-expressing strain, which contrasts with the fluxes during the aerobic part of the fed-batch. The flux from PEP to OAA were consistently higher when $\mathrm{VHb}$ was present in the three experimental conditions tested. This flux may be influenced by a higher AKG demand for the synthesis of heme group (Kwon, de Boer, Petri, \& Dannert, 2003).

Taken together, the presented results demonstrate that VHb expression is an efficient way to enhance highly supercoiled pDNA production in fed-batch cultures under both, aerobic and microaerobic regimes. Although less biomass was attained, more pDNA per biomass unit was obtained when VHb was expressed, which is advantageous for downstream operations. It was found that more pDNA can be synthesized with less RNAII copies/RNAI copies per cell when VHb is expressed. The high lactate accumulation by VHb-expressing cells may be partially explained by insufficient $\mathrm{NAD}^{+}$regeneration in relation with the amount necessary for the increased glycolytic fluxes. Such effects may be overcome by increasing the amount of active VHb or by increasing the flux over the lower part of the TCA.

\section{MATERIALS AND METHODS}

2.1 Bacterial strains, plasmid and precultures. E. coliK-12 derivatives W3110rec - $^{-}$, and W3110recA $-v g b^{+}$were obtained as described elsewhere (Jaén, Velazquez, Delvigne, Sigala, \& Lara, 2019). The strains were transformed with plasmid pUC18 and preserved at $-70{ }^{\circ} \mathrm{C}$. The strains were precultured in lysogenic broth (LB) supplemented with ampicillin disodium salt $(100 \mu \mathrm{g} / \mathrm{ml})$ for 8-10 hr. An aliquot was taken to inoculate $50 \mathrm{~mL}$ of mineral medium. The composition of the mineral medium (in $\mathrm{g} / \mathrm{L}$ ) was as follows: $\mathrm{K}_{2} \mathrm{HPO}_{4}, 17 ; \mathrm{KH}_{2} \mathrm{PO}_{4}, 5.3 ;\left(\mathrm{NH}_{4}\right)_{2} \mathrm{SO}_{4}, 2.5 ; \mathrm{NH}_{4} \mathrm{Cl}, 1.0 ;$ citrate- $\mathrm{Na}_{3} \cdot 2 \mathrm{H}_{2} \mathrm{O}, 2 ; \mathrm{MgSO}_{4} \cdot 7 \mathrm{H}_{2} \mathrm{O}, 1.0$; and thiamine- $\mathrm{HCl}, 0.01$. The medium was supplemented with a trace element solution of $2 \mathrm{~mL} / \mathrm{L}$ and $100 \mu \mathrm{g} / \mathrm{mL}$ ampicillin disodium salt. The trace element solution composition is described elsewhere (Jaén, Velazquez, Delvigne, Sigala, \& Lara, 2019). Glucose was added at a final concentration of $2.5 \mathrm{~g} / \mathrm{L}$. Precultures were performed in baffled shake flasks incubated for $12-16 \mathrm{hr}$ at $250 \mathrm{rpm}$ in a shaker with an orbital diameter of $50 \mathrm{~mm}$. Then, the broth was collected and centrifuged at $7500 \mathrm{rpm}$ for $10 \mathrm{~min}$, and the cell pellet was washed and resuspended in $3 \mathrm{~mL}$ of fresh medium and used to inoculate the main cultures.

2.2 Bioreactor cultures. Bioreactor cultures were carried out in a 1-L Biostat B Plus stirred tank bioreactor (Sartorius BBI, Melsungen, Germany) at $37^{\circ} \mathrm{C}, \mathrm{pH} 7.2$, air flow $1 \mathrm{vvm}$. DOT was measured with an optical sensor (Visiferm 120 Arc, Hamilton, Bonaduz, Switzerland). The batch phase started with $500 \mathrm{~mL}$ of medium supplemented with $15 \mathrm{~g} / \mathrm{L}$ glucose and $100 \mathrm{mg} / \mathrm{L}$ ampicillin disodium salt. The DOT was controlled at $30 \%$ air sat. during the first $6 \mathrm{~h}$ by a PI controller in the agitation cascade mode $\left(t_{i}=50 \mathrm{~s} ; x_{p}=140 \% ; t_{D}=0 \mathrm{~s}\right.$; dead band $=0.1 \%$ ) using MFCS/DA software (Sartorius BBI, Melsungen, Germany). After 6 h of culture, the DOT level was changed to $2 \%$ air sat. The fed-batch phase was started after initial glucose depletion. A concentrated glucose solution $(600 \mathrm{~g} / \mathrm{L})$ supplemented with ampicillin disodium salt was added to the medium using a Watson-Marlow 101U/R programmable peristaltic pump (Watson Marlow, MA, USA). The exponential feeding rate was programmed to maintain the specific growth rate at $0.15 \mathrm{~h}^{-1}$. Off-gas composition was monitored using a BlueInOne Ferm (BlueSens, Herten, Germany) gas analyzer. Oxygen and $\mathrm{CO}_{2}$ evolution rates (OTR and CER respectively) were calculated from the proper mass balances using the off-gas composition (Jaen, Velazquez, Sigala, \& Lara, 2019).

2.3 Off-line analyses. The biomass concentration was determined as the dry cell weight. Glucose and 
ethanol were quantified by an enzymatic-electrochemical method in a YSI-2900 Biochemistry Analyzer (Yellow Spring Instruments, USA). Organic acids were quantified by HPLC (ProStar 210 HPLC system, Agilent Technologies, Santa Clara, CA, USA). Cell lysis and pDNA purification and extraction were carried out using a QIAprep Miniprep Kit (Qiagen, Germany) according to the manufacturer's recommendations. pDNA was quantified by UV spectroscopy in a Nanodrop 2000 spectrophotometer (Thermo Scientific, WI, USA). Monomeric pDNA supercoiled fraction (SCF) was determined by electrophoresis of $100 \mathrm{ng}$ of pDNA in 0.8 $\%$ agarose gel in TAE buffer for $1 \mathrm{~h}$ at $80 \mathrm{~V}$. Restriction analysis was carried out to confirm the plasmid identity using Hind III enzyme.

2.4 PCN, relative RNAII/RNAI and $v g b$ copy number determinations. The PCN was determined through RT-qPCR absolute quantification. Standard curve experiments were executed in a 7500 Real-Time PCR System (Applied Biosystems, CA, USA) using SYBR Green (Applied Biosystems, CA, USA). Plasmid copy quantification was determined using the bla gene from pUC18 and the chromosomal reference gene ihfB . RNA integrity was evaluated by chip microfluidics electrophoresis in a 2100 Bioanalyzer (Agilent CA, USA). The RNA integrity number (RIN) was automatically calculated by the 2100 Expert Software (Agilent, CA, USA). All the samples had RINs between 8 and 9, which are acceptable for our purposes. RNAI and RNAII were determined by RT-qPCR absolute quantification. Standard curve experiments were executed in a 7500 Real-Time PCR System (Applied Biosystems, CA, USA) using SYBR Green. The sequence of primers and reaction conditions are detailed elsewhere (Jaen, Velazquez, Delvigne, Sigala, \& Lara, 2019). The same methods were applied for vgbmRNA quantitation using the primers 5'ATGTTAGACCAGCAAACCATTAACATC-3' (forward) and 5'-TTATTCAACCGCTTGAGCGTACAAATC3' (reverse).

2.5 Flux Balance Analysis. The flux distribution in the metabolic network was calculated based on a linear programming algorithm, using the specific uptake rates $\left(q_{O 2}, q_{S}\right)$ and specific production rates $(\mu$ $\left., q_{C O 2}, q_{b y-p r o d u c t s}\right)$ as inputs for the calculations as detailed previously (Jaén, Olivares, Sigala, \& Lara, 2017). The network comprised 103 reactions and 76 metabolites (intracellular and extracellular) covering glycolysis, PPP, tricarboxylic acids cycle, and mixed-acid fermentation pathways. It included reactions for transport and exchange. Maximization of biomass synthesis was the objective function employed for all cases Simulations were run using MatLab.

\section{REFERENCES}

Ahn, D. G., Shin, H. J., Kim, M. H., Lee, S., Kim, H. S., Myoung, J., Kim, B. T., \& Kim, S. J. (2020). Current Status of Epidemiology, Diagnosis, Therapeutics, and Vaccines for Novel Coronavirus Disease 2019 (COVID-19). Journal of Microbiology and Biotechnology, 30(3),313-324.

Cullis, P.M., Maxwell, A., \& Weiner, D.P. (1992). Energy coupling in DNA gyrase: a thermodynamic limit to the extent of DNA supercoiling.Biochemistry, 31(40), 9642-9646.

del Solar, G., \& Espinosa, M. (2000). Plasmid copy number control: An ever-growing story. Molecular Microbiology, 37, 492-500.

Enayati, N., Tari, C., Parulekar, S. J., Stark, B. C., \& Webster, D. A. (1999). Production of $\alpha$-Amylase in Fed-Batch Cultures of $v g b^{+}$and $v g b^{-}$Recombinant Escherichia coli : Some Observations.Biotechnology Progress, 15(4), 640-645.

Freudenau, I., Lutter, P., Baier, R., Schleef, M., Bednarz, H., Lara, A. R., \& Niehaus, K. (2015). ColE1plasmid production in Escherichia coli : Mathematical simulation and experimental validation. Frontiers in Bioengineering and Biotechnology, 127, 1-14.

Frey, A.D., Fiaux, J., Skyperski, T., Wütrich, K., Bailey, J.E., \& Kallio, P.T. (2001). Dissection of Central Carbon Metabolism of Hemoglobin-Expressing Escherichia coli by ${ }^{13} \mathrm{C}$ Nuclear Magnetic Resonance Flux Distribution Analysis in Microaerobic Bioprocesses. Applied and Environmental Microbiology, 67(2),680-697.

Jaén, K. E., Olivares, R., Sigala, J. C, \& Lara, A. R. (2017). Heterogeneous oxygen availability affects the 
titer and topology but not the fidelity of plasmid DNA produced by Escherichia coli . BMC Biotechnology. $17: 60$.

Jaén, K. E., Velazquez, D., Delvigne, F., Sigala, J. C., \& Lara, A. R. (2019). Engineering E. coli for improved microaerobic pDNA production. Bioprocess and Biosystems Engineering, 42(9),1457-1466.

Jaén, K. E., Velázquez, D., Sigala, J. C., \& Lara, A. R. (2019). Design of a microaerobically inducible replicon for high-yield plasmid DNA production. Biotechnology and Bioengineering. 116(10), 2514-2525.

Kwon, S.J., de Boer, A.L., Petri, R., \& Dannert, C.S. (2003). High-Level Production of Porphyrins in Metabolically EngineeredEscherichia coli : Systematic Extension of a Pathway Assembled from Overexpressed Genes Involved in Heme Biosynthesis. Applied and Environmental Microbiology. 69(8):4875-4883.

Lara, A. R., Jaén, K. E., Folarin, O., Keshavarz-Moore, E., \& Büchs J. (2019). Effect of the oxygen transfer rate on oxygen-limited production of plasmid DNA by Escherichia coli . Biochemical Engineering Journal, 150, 107303.

Ma, C. C., Wang, Z. L., Xu, T., He, Z.Y., \& Wei, Y.Q. (2020). The approved gene therapy drugs worldwide: from 1998 to 2019.Biotechnology Advances, 40 , 107502.

Palomares, L. A., Lara, A. R., \& Ramírez, O.T. (2010). Bioreactor Scale-Down. In: Encyclopedia of Industrial Biotechnology: Bioprocess, bioseparation and cell technology . MC Flickinger (ed). John Wiley and Sons. NY, EUA.

Pablos, T. E., Sigala, J. C., Le Borgne, S., \& Lara AR. (2014). Aerobic expression of Vitreoscilla hemoglobin efficiently reduces overflow metabolism in Escherichia coli . Biotechnology Journal. 9(6) , 791-799.

Passarinha, L. A., Diogo, M. M., Queiroz, J. A., Monteiro, G. A., Fonseca, J. P., \& Prazeres, D. M. F. (2006). Production of ColE1 type plasmid by Escherichia coli DH5 $\alpha$ cultured under nonselective conditions. Journal of Microbiology and Biotechnology, 16,20-24.

Roca, A.I., \& Cox, M.M. (1997). RecA protein: structure, function, and role in recombinational DNA repair. Progress in Nucleic Acid Research and Molecular Biology 56 , 129-223.

Rozkov, A., Larsson, B., Gillström, S., Björnestedt, R., \& Schmidt, S.R. (2008). Large-scale production of endotoxin-free plasmids for transient expression in mammalian cell culture. Biotechnology and Bioengineering, 99(3) , 557-566.

Stark, B. C., Pagilla, K. R., \& Dikshit, K. L. (2015) Recent applications of Vitreoscilla hemoglobin technology in bioproduct synthesis and bioremediation. Applied Microbiology and Biotechnology, 99, 1627-1636.

Veeravalli, K., Schindler, T., Dong, E., Yamada, M., Hamilton, R., \& Laird, M. W. (2018). Strain engineering to reduce acetate accumulation during microaerobic growth conditions in Escherichia coli .Biotechnology Progress, 34, 303-314.

Wehrs, M., Tanjore, D., Eng, T., Lievense, J., Pray, T.R., \& Mukhopadhyay, A. (2019). Engineering Robust Production Microbes for Large-Scale Cultivation. Trends in Biotechnology, 27(6), 524-537.

\section{Figure legends.}

Figure 1. Production of pUC18 in E. coliW3110recA - (left panel) and W3110recA - vgb ${ }^{+}$(right panel) in fed-batch cultures with a step-change to $\mathrm{O}_{2}$-limited regime. The time profiles of biomass and glucose concentrations (a, e), organic acids and ethanol accumulation (b, f), plasmid DNA (pDNA) production, supercoiled pDNA fraction, and pDNA yield from biomass $(\mathrm{c}, \mathrm{g})$, oxygen transfer rate (OTR), carbon dioxide evolution rate (CER) and dissolved oxygen tension (DOT) $(\mathrm{d}, \mathrm{h})$ are shown. Error bars show the deviation between duplicates. pDNA, plasmid DNA.

Figure 2. (a) Plasmid copy number per chromosome (PCN), (b) Relative RNAII copies/RNAi copies, normalized to sample at $4 \mathrm{hr}$, and (c) relative $v g b$ copy number, normalized to sample at $4 \mathrm{hr}$, in fed-batch 
culture samples taken at 4,10 and $12 \mathrm{hr}$ of culture.

Figure 3. Metabolic fluxes estimated by flux balance analysis in fed-batch cultures of W3110rec $A^{-}$(left row) and $\mathrm{W} 3110 \mathrm{rec} A^{-} v g b^{+}$(right row) with a step-change to $\mathrm{O}_{2}$-limited regime. Calculations were performed for pseudo steady-states (constant growth rates and yields) during the aerobic batch (top numbers), aerobic phase of the fed-batch (middle numbers) and microaerobic phase of the fed-batch (bottom numbers). All values are given in $\mathrm{mmol} / \mathrm{g} \mathrm{hr}$.
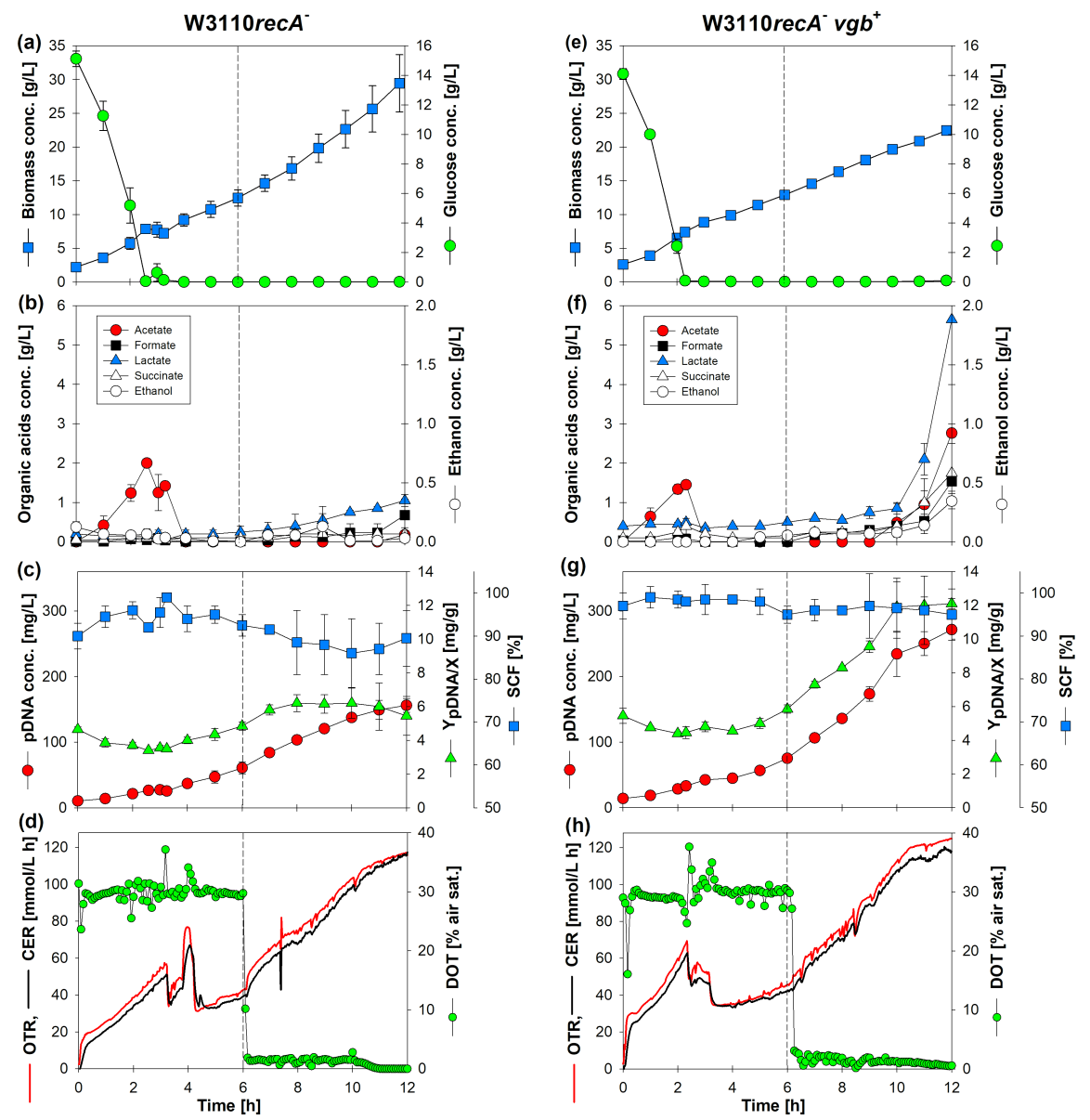
(a)



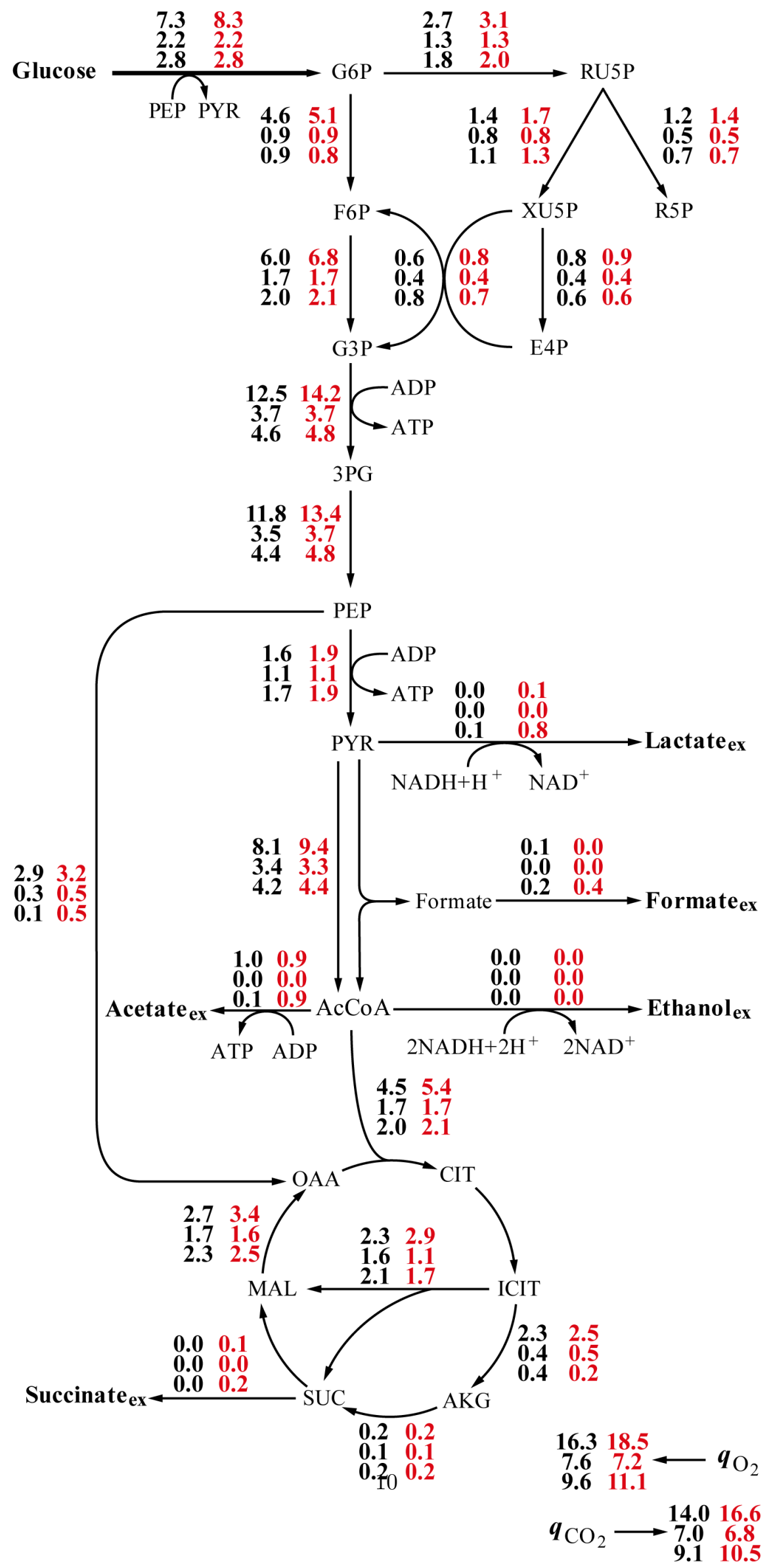

